\title{
Actualización en radiología dental. Radiología convencional Vs digital
}

\author{
Barbieri Petrelli G*, Flores Guillén J*, Escribano Bermejo $M^{* *}$, Discepoli $N^{* * *}$
}

\section{RESUMEN}

Desde su incorporación a la práctica odontológica la radiología digital ha experimentado un importante desarrollo. El continuo avance de las tecnologías en las que se sustenta ha dotado a estos sistemas de interesantes prestaciones que pueden facilitar el diagnóstico y manejo de imágenes radiográficas. Con estos avances la radiología digital ha despertado un interés creciente entre los profesionales de la odontología, especialmente durante los últimos años, en los que ha aumentado notoriamente tanto la cantidad de sistemas comercializados como el número de odontólogos que han decidido sustituir la radiología convencional por un sistema digital en sus clínicas. En esta revisión de la literatura se tratará de ofrecer una visión actualizada de los distintos sistemas disponibles en la actualidad, así como una comparativa entre ellos basada en estudios publicados recientemente.

Palabras clave: Radiología digital directa, radiología digital indirecta, resolución.

\section{SUMMARY}

Since its appearance in dentistry, digital radiology has experienced a notorious development. A constant advance in the corresponding technologies has enhanced these digital systems with interesting features which may facilitate radiological diagnosis and image management. Due to these advances digital radiology has elicited a growing interest in dentistry. Specially during the last years there has been an increase both in the amount of available systems and in the number of dentists who have incorporated a digital system in their offices. This literature review aims to offer an updated view of the available systems and a comparison of them based in recent studies.

Key words: Direct digital radiography, indirect digital radiography, resolution.

Aceptado para publicación: Mayo 2005.

* Alumno Master de Periodoncia UCM.

** Doctorando UCM.

*** Licenciado en odontología por la universidad de Siena.

Barbieri Petrelli G, Flores Guillén J, Escribano Bermejo M, Discepoli N. Actualización en radiología dental. Radiología convencional Vs digital. Av. Odontoestomatol 2006; 22-2: 131-139.

\section{INTRODUCCIÓN}

Durante la década pasada la radiología digital fue introducida en la práctica odontológica. A mediados de los 90 la baja resolución de estos sistemas limitó en gran medida su aplicación en odontología. Sin embargo al final de la década los avances tecnológicos supusieron una drástica mejora en las posibili- 
dades diagnósticas de estos sistemas de radiología digital. Hoy en día estos avances incluyen la simplificación tanto de los aparatos como de los programas informáticos a los que van asociados, una rápida obtención de la imagen radiográfica, grandes prestaciones en el tratamiento de dichas imágenes y, en definitiva, mayores comodidades tanto para el dentista como para el paciente. De este modo la aceptación de la radiología digital ha ido creciendo en el mundo de la odontología y cada año son más los profesionales que deciden incorporar esta tecnología en sus clínicas.

Por otra parte, a lo largo de esta evolución ha ido aumentando la oferta de estos sistemas en el mercado y hoy en día disponemos de numerosos aparatos de radiología digital entre los que elegir.

En este contexto, el objetivo de este artículo es, por una parte, facilitar un acercamiento de esta tecnología a aquellos profesionales que no estén familiarizados con ella y, por otra parte, ofrecer una actualización del tema, así como una comparativa basada en la literatura entre los distintos sistemas, incluida la radiología convencional. Se pretende de este modo esclarecer, a modo de conclusión, las ventajas e inconvenientes que presentan estos sistemas.

\section{Tipos de radiología digital}

Existen actualmente dos tecnologías diferentes en radiología digital. Para evitar el uso de nombres comerciales emplearemos los siguientes términos: radiología digital directa (RDD) y radiología digital indirecta (RDI).

\section{Radiología digital directa}

Emplea como receptor de rayos $\mathrm{X}$ un captador rígido habitualmente conectado a un cable a través del cual la información captada por el receptor es enviada al ordenador. Se denomina directa porque, a la inversa de la indirecta, no requiere ningún tipo de escaneado tras la exposición a los rayos $\mathrm{X}$, sino que el propio sistema realiza automáticamente el proceso informático y la obtención de la imagen.

\section{Radiología digital indirecta (radiología con fósforo fotoestimulable)}

La imagen es capturada de forma analógica en una placa de fósforo fotoestimulable y convertida en digital tras su procesado o escaneado.

\section{Principios básicos}

\section{Radiología digital directa}

Funciona con sensores fotosensibles similares a los de las cámaras fotográficas digitales. Puesto que estos sensores se estimulan con luz y se deteriorarían al ser expuestos a rayos $\mathrm{X}$, el receptor o captador de estos sistemas consta de otros dos componentes, además del sensor (Figura 1). La primera capa, el escintilador, se encarga de transformar los rayos $\mathrm{X}$ en luz. Una pequeña cantidad de radiación atraviesa el escintilador sin ser convertida en luz, por lo que una segunda capa compuesta por fibra óptica $\mathrm{u}$ otros materiales evita la penetración de los rayos $\mathrm{X}$ hasta el sensor y por tanto su deterioro.

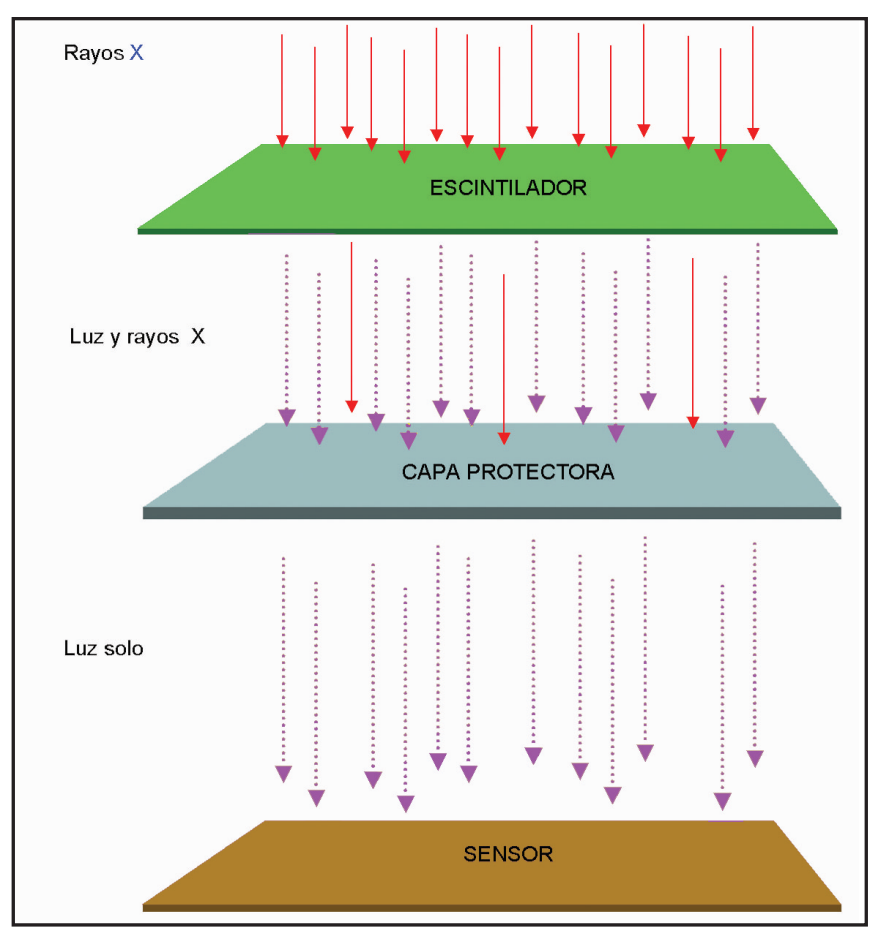

Fig. 1. Estructura de un captador de radiología digital directa. 
El sensor esta formado por una estructura de celdillas o píxeles fotosensibles capaces de almacenar fotones, y que convierten la señal luminosa que reciben en una señal eléctrica de intensidad proporcional. Esta señal eléctrica es enviada a un conversor analógico digital o DAC que, como su propio nombre indica, transforma la señal analógica (eléctrica) en una digital (basada en un código binario). De este modo, la señal luminosa que recibe cada píxel del sensor será convertida en un valor formado por ceros y unos, y este valor será interpretado como un determinado nivel de gris. La unión de todos los puntos grises correspondientes a las distintos píxeles generará finalmente una imagen.

\section{Radiología digital indirecta}

Emplea placas de aspecto similar a las películas radiográficas convencionales pero compuestas por una emulsión cristalina de fluorohaluro de bario enriquecido con Europio. Esta emulsión es sensible a la radiación. Los rayos $\mathrm{X}$ provocan la excitación y liberación de un electrón del Europio, que es captado por una vacante halógena del fósforo de almacenamiento. Las vacantes electrónicas y los electrones captados se recombinan y causan luminiscencia, convirtiendo los rayos $\mathrm{X}$ en energía latente almacenada. Un láser de helio-néon estimula la luminiscencia de la placa, liberando los electrones atrapados, que se recombinan con las vacantes del Europio. La energía, en forma de luz, es captada por un tubo fotomultiplicador y transformada en señal eléctrica. Finalmente, la señal resultante es convertida en digital mediante un conversor analógico-digital, que determina el número máximo de tonos de gris.

\section{Características técnicas (resolución)}

En la actualidad existen tres tipos de sensores empleados en RDD:

- CCD (charge-couple device).

- CMOS-APS (complementary metal oxide semiconductor active pixel sensor).

- Super CMOS.
Estos sensores tienen distintas características y propiedades y, por tanto, confieren diferentes prestaciones al sistema de RDD. Los CCD tienen una mayor sensibilidad a la luz y proporcionan imágenes de más calidad, pero tienen también un coste más elevado. Los CMOS-APS son externamente idénticos a los CCD pero utilizan una nueva tecnología en píxeles (APS). Ofrecen las siguientes ventajas sobre los CCD:

- Reducen 100 veces los requisitos del sistema para procesar la imagen.

- Mejora la fiabilidad y la vida media de los sensores.

- Capacidad de transmisión en cada una de las celdas. Esto evita el efecto de «blooming» o de contaminación entre píxeles vecinos cuando hay situaciones de sobreexposición.

- Permite mejores opciones de interpolación de la imagen.

- Más fáciles de interconectar a nuevos sistemas que los CCD.

Por otra parte tienen también algunas desventajas:

- Son menos sensibles y de menor calidad, pero al ser fáciles de fabricar son más baratos.

- Son muy sensibles al ruido de imagen, tienen poca sensibilidad.

- El área activa de estos sensores es más pequeña.

Por último, el Super CMOS es una evolución del CMOS que según sus fabricantes ofrece una resolución superior.

La resolución espacial, medida en pares de líneas/ $\mathrm{mm}$, representa la fidelidad de la imagen en cuanto a su capacidad para mostrar detalles más pequeños (Figura 2).

Las placas radiológicas convencionales tienen una resolución superior a $20 \mathrm{pl} / \mathrm{mm}$ (1). Algunos sistemas de RDD alcanzan esta resolución, mientras que los de RDI pueden llegar a 12,5 pl/mm, como en el caso de Digora, que anteriormente era de 6-8 pl/ $\mathrm{mm}$. Todo esto adquiere valor al compararlo con la capacidad que tiene el ojo humano para distinguir pares de líneas, llegando el mismo a una resolución de $8-10 \mathrm{pl} / \mathrm{mm}$. 


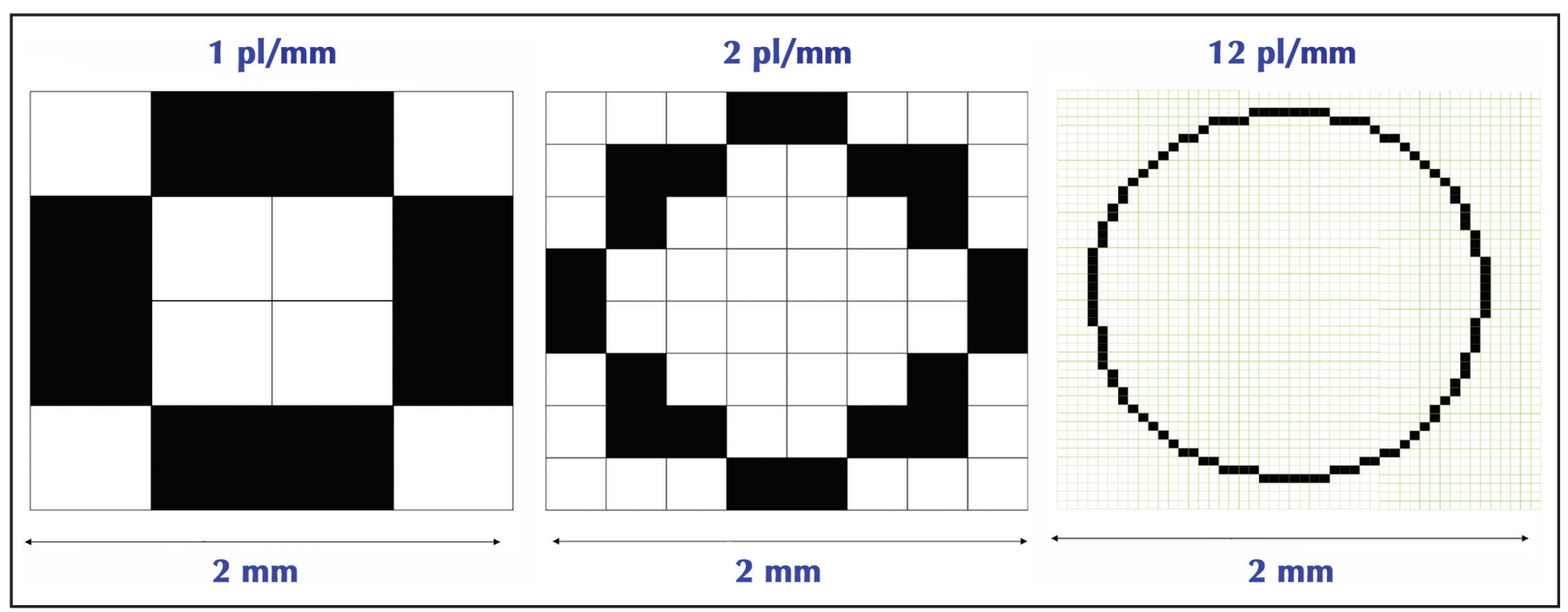

Fig. 2. Representación de distintas resoluciones.

A este respecto, Mol (2), concluyó que "la resolución de la radiografía digital es similar o inclusive peor que la radiografía convencional, lo cual no implica una mejora o empeoramiento de la efectividad diagnóstica".

\section{SISTEMAS COMERCIALIZADOS. DESCRIPCIÓN Y DIFERENCIAS}

\section{Radiología digital directa}

En la actualidad, son ya muchas las casas comerciales que han desarrollado sistemas de radiología digital directa. Algunas incluso han lanzado al mercado varios tipos o categorías con diferentes prestaciones. Se comercializan, por tanto, un buen número de sistemas. Algunos ejemplos de los más conocidos en España son los comercializados por las casas Kodak (Figura 3), Gendex, o Sirona, entre otros. El manejo de todos ellos es similar: primero se coloca el captador en una funda desechable (Figura 4) para evitar infecciones cruzadas. Entonces es colocado en boca del mismo modo que si se tratara de una película radiográfica y se procede a la exposición a rayos $\mathrm{X}$. En unos pocos segundos la imagen aparece en el monitor del ordenador.

En cuanto a las características y prestaciones de los distintos sistemas, podemos encontrar una cierta variedad entre ellos. La guía de técnicas y productos dentales CRA Newsletter publicó en febrero de 2005 una completa comparativa entre ocho sistemas de radiología digital (siete de RDD y uno de RDI) a partir de los resultados de una serie de 500 encuestas realizadas a dentistas (3). Las encuestas evaluaban la apreciación del dentista acerca de características como el tamaño del receptor, posibilidad de paralelizar, comodidad en el uso del receptor y en su recambio, medidas higiénicas, sencillez de manejo, calidad de imagen, herramientas para el tratamiento

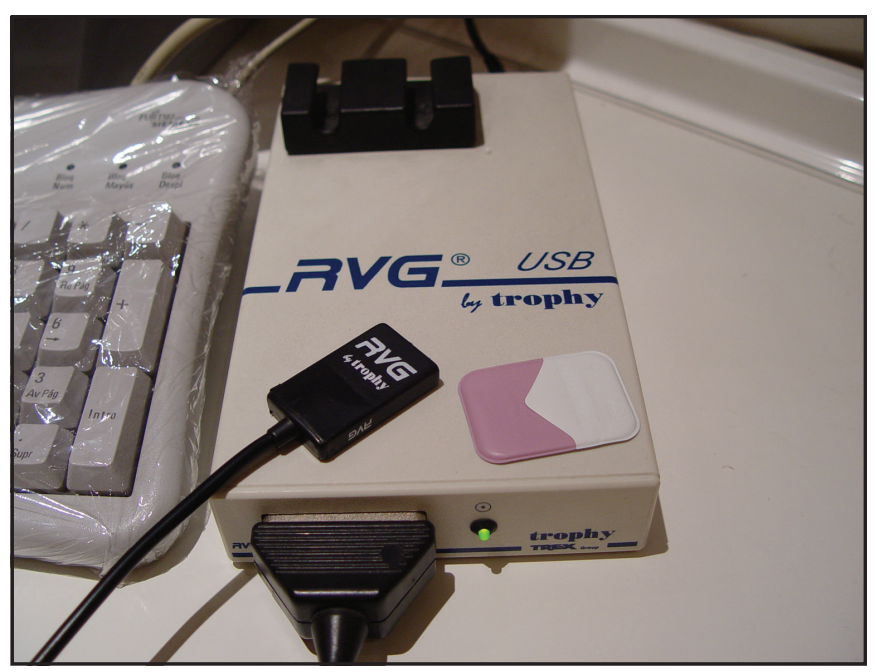

Fig. 3. RDD (RVG). 


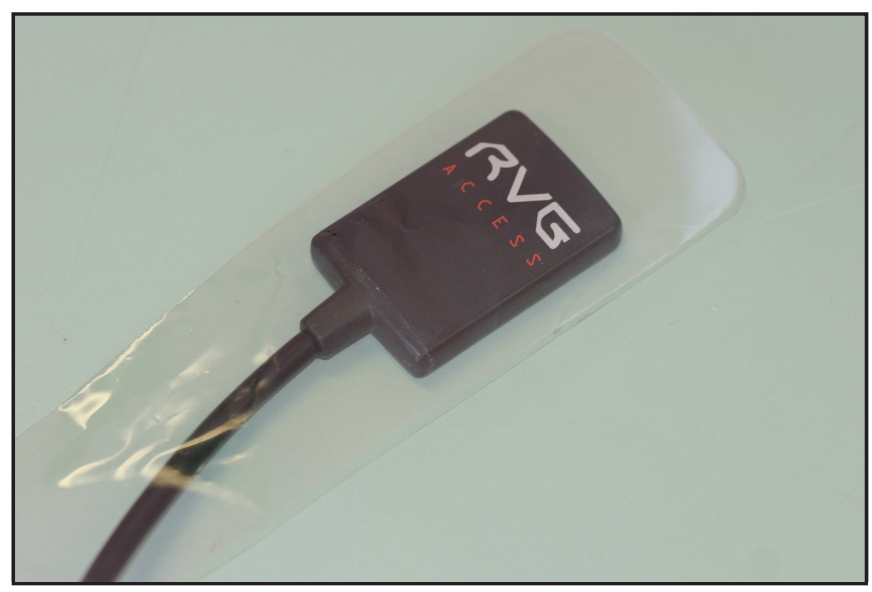

Fig. 4. Captador con funda desechable.

de imágenes, etc. Las valoraciones globales obtenidas fueron:

- Buena para los sistemas de RDD Sidexis IO2 de la casa Sirona, y Lightyear de Lightyear Technology, y sistema de RDI ScanX de la casa Air Techniques.

- Excelente para los sistemas de RDD Kodak RVG 6000 (Kodak Dental Systems Group), Dexis (Dexis Digital X-Ray), Image RAYi (Dentrix), CDR (Schick Technologies) y el sistema de RDD con captador inalámbrico CDR Wireless (Schick Technologies).

El sistema Kodak RVG 6000 obtuvo la puntuación más alta.

\section{Radiología digital indirecta}

En la actualidad, podemos encontrar diferentes sistemas de placas de fósforo en el mercado:

- Digora (Soredex, Helsinki, Finland).

- Cd-dent (Antes Digi-Dent, Orex, Yokneam, Israel).

- DenOptix (Gendex, Dentsply, Milan, Italy).

Básicamente, todos los sistemas constan de una serie de receptores de fósforo con diferentes formas y tamaños, y con capacidad de flexión (figura 5). Estas placas receptoras se colocarán en unas fundas pro-

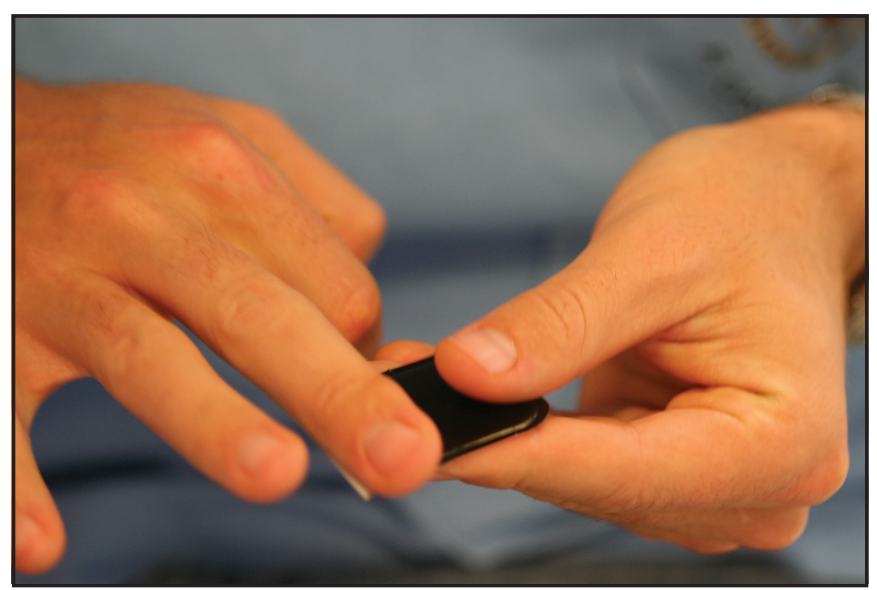

Fig. 5. Placa de fósforo fotoestimulable.

tectoras que se desecharán tras su utilización. Una vez tomada la radiografía y desechada la funda protectora, la placa se colocará en el escáner (figura 6) que leerá la imagen tomada, la transmitirá al ordenador y, finalmente, borrará la imagen para permitir la nueva utilización del receptor.

Durante todo este proceso de manipulación pueden producirse alteraciones, tanto de la imagen como de los propios receptores. En la literatura podemos encontrar diferentes artículos que nos lo demuestran.

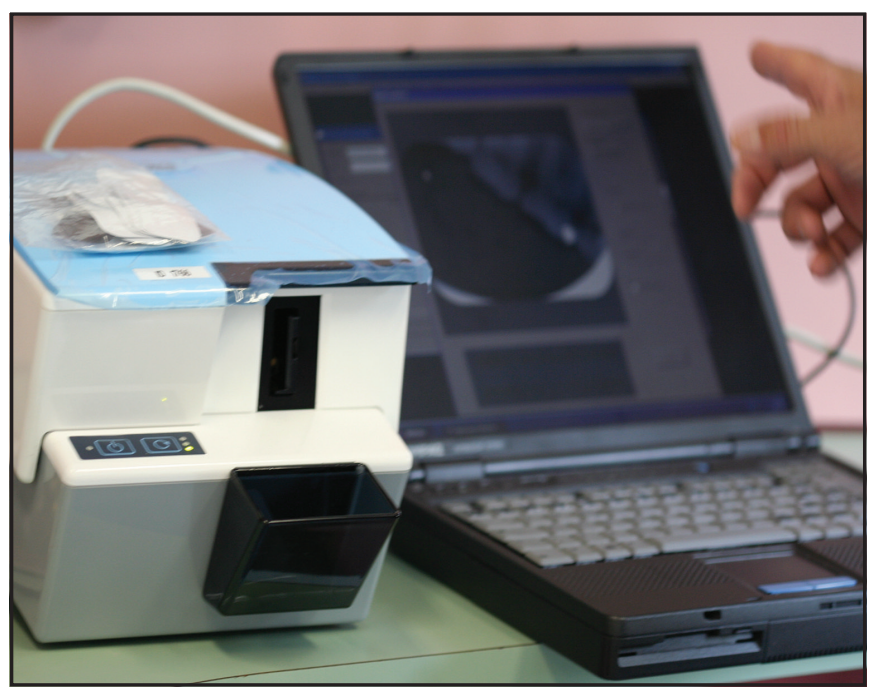

Fig. 6. Escáner del sistema Digora ${ }^{\circledR}$. 
Bedard y cols. (4) realizaron un trabajo en el que el objetivo era evaluar la durabilidad de las placas de fósforo fotoestimulable y determinar los factores que pueden contribuir a una posible menor durabilidad de las mismas. Entre las posibles causas que pueden producir el deterioro de la placa, destacaron: colocación en barrera protectora, colocación en paralelizador, colocación en otro soporte, colocación en boca y colocación en el tambor del escáner. Estudiantes de odontología realizaron 821 imágenes digitales, y 5 radiólogos fueron los encargados de determinar si estas imágenes eran o no compatibles para diagnóstico. Los resultados mostraron que el 95\% de las imágenes no eran aceptables tras la utilización de las placas de fósforo durante aproximadamente 50 exposiciones.

Estos resultados hay que interpretarlos dentro del contexto en el que se encuentran. En primer lugar, son estudiantes $\mathrm{y}$, por consiguiente, con menos experiencia. Y en segundo lugar, la mayor parte de las alteraciones de las placas se producían durante la colocación de las mismas en el tambor del escáner, proceso muy simplificado en la actualidad.

Ramamurthy y cols. (5) determinaron que la luz ambiental aumenta el ruido de la imagen debido a que puede borrar de forma parcial la imagen latente. Debido a que no se considera buena práctica compensar este fenómeno aumentando la exposición, es preferible la reducción de la luz ambiental.

Martins y cols. (6) publicaron un estudio en el que el objetivo era evaluar el efecto de diferentes condiciones y tiempos de almacenamiento en placas de fósforo fotoestimulable. El estudio se realizó en mandíbulas disecadas y los sistemas utilizados fueron el Digora ${ }^{\circledR}$ y Den Optix ${ }^{\circledR}$. Las placas fueron almacenadas bajo 3 condiciones diferentes: Temperatura ambiente $\left(23,3{ }^{\circ} \mathrm{C}\right.$ y $56,5 \%$ humedad), frigorífico $\left(8,2{ }^{\circ} \mathrm{C}\right.$ y $43 \%$ humedad) y baja humedad (24,5 ${ }^{\circ} \mathrm{C}$ y $25 \%$ humedad). El escaneado de las mismas se realizó a las 6 h, 12 h, 18 h, 24 h, 48 h y 72 h. Con el sistema Den Optix ${ }^{\circledR}$ no se encontraron diferencias, mientras que con el Digora ${ }^{\circledR}$ aparecieron diferencias con el gold estándar (escaneado inmediato) tras $12 \mathrm{~h}$ (frigorífico) y tras $24 \mathrm{~h}$ ( $\mathrm{T}^{\mathrm{a}}$ ambiente o baja humedad).

\section{DIFERENCIAS BASADAS EN LA LITERATURA}

\section{RDI Vs convencional}

Hintze y cols. (7) realizaron en 2002 un estudio con el objetivo de evaluar la precisión en la detección de caries de una película convencional y 4 sistemas de RDI utilizados con 2 tiempos de exposición diferentes ( $10 \%$ y $25 \%$ de la correspondiente a la película). Los sistemas utilizados fueron los siguientes: sistemas de fósforo fotoestimulable Den Optix ${ }^{\circledR}, \mathrm{Cd}$ dent $^{\circledR}$, Digora blue ${ }^{\circledR}$ y Digora white $^{\circledR}$, y película Ektaspeed plus ${ }^{\circledR}$. Cuatro observadores utilizaron una escala de 0-5 en función de la detección o no de la caries $(1=$ no presente, $2=$ probablemente no presente, $3=$ inseguro, $4=$ probablemente presente, $5=$ presente) en 190 dientes extraídos. Según sus resultados la precisión de Cd-dent ${ }^{\circledR}$ fue estadísticamente menor para caries proximales con exposición del $25 \%$. Digora blue ${ }^{\circledR}$ fue el mejor sistema digital para caries oclusales con $25 \%$ del tiempo de exposición.

El tiempo de exposición influyó para la detección de:

- Caries proximal con los sistemas Den Optix ${ }^{\circledR}$ Digora ${ }^{\circledR}$ blue.

- Caries oclusal con Digora blue ${ }^{\circledR}$.

Los autores concluyeron que con estos sistemas de $\mathrm{RDI}$ parece conveniente reducir la exposición en un $75 \%$ respecto a la película convencional.

Kaeppler y cols. (8) compararon también un sistema de RDI con una película radiográfica con los siguientes objetivos:

1. Comparar placas de fósforo fotoestimulable (Digora $\left.{ }^{\circledR}\right)$ con radiografía convencional en cuanto a precisión de medidas lineales del hueso alveolar.

2. Comparar estos sistemas en cuanto a la capacidad de distinguir diferentes estructuras anatómicas.

Para ello emplearon la siguiente metodología:

- Para el objetivo 1, un examinador realizó mediciones en 108 pares de radiografías tomadas en 
mandíbulas disecadas, valiéndose de la colocación de pins metálicos a $10 \mathrm{~mm}$ de distancia del hueso marginal.

- Para el objetivo 2, dos examinadores determinaron la capacidad de visualizar el ligamento periodontal, hueso periapical y cresta alveolar mediante una escala de valores $(1=$ bien, $2=$ satisfactorio, $3=$ pobre); en 51 pares de radiografías tomadas a 21 pacientes.

Obtuvieron los siguientes resultados:

- Objetivo 1: La exactitud de las mediciones lineales fue mayor con el sistema Digora ${ }^{\circledR}$, y la desviación media fue del $1 \%$ (digital) y 3,9\% (convencional).

- Objetivo 2: La radiografía convencional fue capaz de distinguir las estructuras anatómicas de igual forma o incluso mejor que la radiografía digital.

En 2005, Akdeniz y Sogur (9) compararon subjetivamente dos películas convencionales y una digital (Digora ${ }^{\circledR}$ ) respecto a la longitud y homogeneidad en tratamientos endodónticos.

Se realizaron tratamientos endodónticos en 20 molares inferiores extraídos para, posteriormente, realizar la toma de las radiografías correspondientes. Las radiografías convencionales se observaron sin magnificación. En el caso de las digitales, éstas fueron examinadas de dos formas diferentes; sin modificación alguna o modificando brillo y contraste por parte del examinador $(n=10)$. Se obtuvieron mejores resultados con Digora ${ }^{\circledR}$ modificado por el examinador. El orden de mejor a peor resultado fue el siguiente: Digora modificado $>$ placa E-speed $>$ placa F-speed > Digora.

\section{RDI Vs RDD Vs convencional}

En 2005 Bhaskaran y cols. (10) publicaron un estudio cuyo objetivo fue medir y comparar la calidad de imagen y exposición a rayos $\mathrm{X}$ de tres tipos de sistemas de radiografía intraoral: 1) Película convencional: Kodak F-speed; 2) RDI: Digora FMX; 3) Sistema basado en CCD: Visualix USB. Se realizaron exposi- ciones de entre 10 y 2000 milisegundos, en molares superiores e inferiores de maxilares y mandíbulas disecadas. Las imágenes no fueron reajustadas pero sí clasificadas en función a la calidad a la hora de observar la anatomía del conducto radicular, obturación endodóntica, espacio del ligamento periodontal, lámina dura y detalle del hueso periapical. (Rango de clasificación entre 0 y 4). Según sus resultados la máxima calidad de imagen sólo se observó con película convencional, mientras que ambos sistemas digitales obtuvieron una puntuación máxima de 3.1. La reducción de la dosis de rayos X para obtener máxima calidad con radiología digital fue del $20 \%$ para el Visualix USB y del $70 \%$ para el Digora FMX. Considerando como "aceptable calidad de imagen" a aquellas clasificadas en un rango entre 2 y 4, la mínima dosis aceptable para el Visualix USB fue de un $50 \%$ de reducción sobre la dosis convencional. Digora demostró una mayor latitud o rango de exposición.

\section{RDI Vs RDD}

Wenzel y Kirkevang (11) compararon la exactitud diagnóstica de un sistema de "alta resolución" basado en un sensor CCD y un sistema de "resolución media" basado en placas de fósforo fotoestimulable para detectar fracturas radiculares experimentales.

Los sistemas evaluados fueron Radiovisiografía ${ }^{\circledR}$ O RVG (RDD) y Digora ${ }^{\circledR}$ (RDI). La toma de las radiografías se realizó con diferentes proyecciones (una ortogonal, una con un ángulo vertical de $15^{\circ}$ y dos excéntricas con ángulo horizontal de $15^{\circ}$ ). Tres observadores fueron los encargados de evaluar las distintas radiografías. Según sus resultados, RVG mostró mayor sensibilidad que el Digora. No obstante, deben analizarse estos resultados con cautela. El autor parece decantarse por la RVG desde el principio, puesto que ya en el título del artículo se refiere a ésta como sistema de alta resolución y a Digora como sistema de resolución media. Profundizando un poco más en los resultados podemos observar que las diferencias son estadísticamente significativas sólo en el caso de proyecciones verticales, problema que normalmente se soluciona con la utilización de paralelizadores. 


\section{CONCLUSIONES: VENTAJAS E INCONVENIENTES}

\section{Digital Vs convencional}

\section{- Ventajas}

1. Reducción dosis de exposición

2. Eliminación procesado químico

3. Obtención rápida de la imagen

4. Reutilización

5. Almacenamiento

6. Tratamiento imagen

- Desventajas

1. Coste económico

2. Manipulación cuidadosa

3. No valor jurídico

4. "Control de la infección"

5. "Resolución inferior"

\section{RDI respecto a RDD}

\section{- Ventajas}

1. Ausencia de cable ${ }^{1}$

2. Flexibilidad del receptor

3. Menor grosor de placa

4. Mayor amplitud de exposición

5. "Menor radiación" 2

6. Mayor similitud con convencional

7. Mayor variedad de formas y tamaños

8. Facilidad para paralelizar

- Desventajas

1. Coste económico

2. Menor resolución

3. Necesidad de escáner

4. "Tiempo de procesado"3

${ }^{1}$ Hoy en día se comercializan ya sistemas de RDD sin cable. Tienen el inconveniente añadido de la necesidad de pilas.

2 La dosis de radiación requerida varía entre los distintos estudios revisados. En cualquier caso ambos sistemas utilizan una dosis de radiación claramente inferior a la radiología convencional (12).

3 Actualmente, sistemas como el Digora han reducido el tiempo de escaneado de 20" a tan solo 4". Los sistemas de RDD tardan en procesar la imagen prácticamente lo mismo que los sistemas más rápidos de RDI pero, los primeros, siguen teniendo la ventaja de no necesitar de un escáner, cuya utilización implica una mayor demora en la obtención final de la imagen.

\section{BIBLIOGRAFÍA}

1. Farman AG, Farman TT. A comparison of 18 different X-ray detectors currently used in dentistry. Oral Surg Oral Med Oral Pathol Oral Radiol Endod 2005;99:485-9.

2. Mol A. Imaging methods in periodontology. Periodontol 2000. 2004;34:34-48.

3. Digital radiography-2005 (CRA status report). CRA Newsletter 2005. Feb Vol 29. Issue 2.

4. Bedard, Davis, Angelopoulos. Storage Phosphor Plates: How Durable are they as a Digital Dental Radiographic System?. J Contemp Dent Pract. 2004 May 15;5(2):57-69.

5. Ramamurthy R, Canning CF, Scheetz JP, Farman AG. Impact of ambient lighting intensity and duration on the signal-to-noise ratio of images from photostimulable phosphor plates processed using DenOptix and ScanX systems. Dentomaxillofac Radiol. 2004 Sep;33(5):307-11.

6. Martins MG, Haiter Neto F, Whaites EJ. Analysis of digital images acquired using different phosphor storage plates (PSPs) subjected to varying reading times and storage conditions". Dentomaxillofac Radiol 2003 May;32(3):186-90.

7. Hintze H, Wenzel A, Frydenberg M. Accuracy of caries detection with four storage phosphor systems and E-speed radiographs. Dentomaxillofac Radiol. 2002 May;31(3):170-5.

8. Kaeppler G, Vogel A, Axmann-Krcmar D. Intraoral storage phosphor and conventional radiography in the assessment of alveolar bone structures. Dentomaxillofac Radiol. 2000 Nov;29 (6):362-7.

9. Akdeniz BG, Sogur E. An ex vivo comparison of conventional and digital radiography for perceived image quality of root fillings. Int Endod J. 2005 Jun;38(6):397-401.

10. Bhaskaran V, Qualtrough AJ, Rushton VE, Worthington HV, Horner K. A laboratory com- 
parison of three imaging systems for image quality and radiation exposure characteristics. Int Endod J. 2005 Sep;38(9):645-52.

11. Wenzel A, Kirkevang LL. High resolution chargecoupled device sensor vs. medium resolution photostimulable phosphor plate digital receptors for detection of root fractures in vitro. Dent Traumatol. 2005 Feb;21(1):32-6.

12. Berkhout WE, Beuger DA, Sanderink GC, van der Stelt PF. The dynamic range of digital radiographic systems: dose reduction or risk of overexposure? Dentomaxillofac Radiol. 2004 Jan;33(1):1-5. 